\title{
Developing the phenomenological equations triaxial deformation of concrete under dynamic loads
}

\author{
Mikhail Berlinov, ${ }^{1, *}$ \\ ${ }^{1}$ Moscow State University of Civil Engineering, Yaroslavskoe shosse, 26, Moscow, 129337, Russia
}

\begin{abstract}
The basic assumptions and hypotheses construction of the computational model studies, taking into account the peculiarities of the work force imperfections of materials under dynamic loading, based on the phenomenological laws of nonlinear rheology and deformable elasticcreeping body. The values for the coefficient vibrocreep computational model under triaxial stress-strain state on the basis of the hypothesis of central symmetry of the hysteresis loop. The basic phenomenological equations allow the calculation of concrete elements in the conditions of triaxial stress-strain state under dynamic impacts and taking into account the non-linearity of the rheology of deformation.
\end{abstract}

\section{Introduction}

Intensification creep properties under long-term high-frequency alternating force effects acting in the presence of long-term permanent static loads, called vibrocreep[1] characteristic of many materials, including concrete and steel, which are the main constituents of concrete and soil base upon which all, without exception, buildings and facilities, vibrocreep concrete due to a weakening dynamic intercrystalline bonds in the cement stone and aggregate near the boundary zones by the gradients of the inertial forces and vibration phenomenon thixotropic gel and a structural component[2,3].

Evaluation vibrocreep materials included in the calculation model is based on the following hypotheses.

With long-term exposure to a steady vibratory chart stress- deformation under load and unload each oscillation cycle forms a closed loop (Fig.1).

The area of this loop is a function of the dynamic stress level and does not depend on the oscillation frequency[4].

As shown by numerous experimental data [5], the deformation curves vibrocreep in the presence of long-term static loading affinity similar creep strain curve at said static load. In other words, all the curves of deformation vibrocreepaffinity similar, and the values of their ordinates differ from each other only by certain factors like, called us vibrocreepcoefficients[6].

*Corresponding author: berlinov2010@mail.ru 


\section{Results section}

Mathematical record hypothesis on frequency-independent internal friction as follows:

$$
\begin{gathered}
\frac{\partial}{\partial \omega} \Delta W=0, \\
\Delta W=\varphi(n) \Delta W_{0}, \\
\varphi(n)=\frac{\Delta W_{\infty}}{\Delta W_{0}}+\left(1-\frac{\Delta W_{\infty}}{\Delta W_{0}}\right) e^{-\alpha n},
\end{gathered}
$$

here: $\Delta \mathrm{W}$-area of the hysteresis loop in the coordinate system $\sigma\left|\mathrm{O}^{\mid} \varepsilon^{\mid}\right|$(Fig.1.); $\omega$ - circular frequency; $\Delta \mathrm{W}_{0^{-}}$reversible part of the energy corresponding to the part of the restored work on reversible deformations (area figure $\mathrm{O} A C D$ in Figure 1.), With $\mathrm{n}_{0}$ (n -number cycle fluctuations); $\Delta \mathrm{W}_{\infty}$ - the same for sufficiently large $\mathrm{n} ; \varphi(\mathrm{n})$ - a function of the impact of non-cycle fluctuations.

Dividing, instantaneous deformation and creep deformation:

$$
\Delta W_{0}=\Delta W_{m}+\Delta W_{p}
$$

You can obtain the following relationship:

$$
\Delta W_{0}=\int_{\sigma_{\min }}^{\sigma_{\max }} \bar{\varepsilon}_{m} d \sigma-\int_{\sigma_{\min }}^{\sigma_{\max }} \vec{\varepsilon}_{m} d \sigma+\int_{\sigma_{\min }}^{\sigma_{\max }} \bar{\varepsilon}_{p} d \sigma-\int_{\sigma_{\min }}^{\sigma_{\max }} \vec{\varepsilon}_{p} d \sigma ;
$$

where: $\leftarrow$ and $\rightarrow$ icons denoting respectively loading and unloading; $\varepsilon$ - the equation of the mechanical state of the material (complete relative deformation); $\Delta \mathrm{W}_{\mathrm{m}} \mathrm{m}$ and $\Delta \mathrm{W}_{\mathrm{p}}$ - parts of the hysteresis loop, corresponding instantaneous deformation and creep deformation.

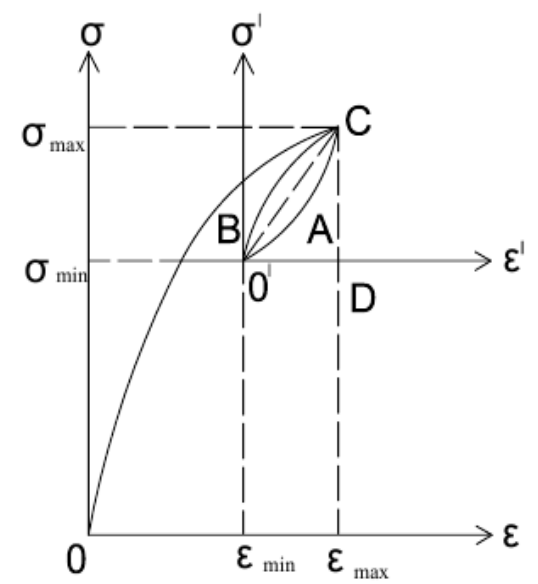

Fig. 1. Hysteresis loop under dynamic loads

Properties of materials considered in this study are subject to the laws of phenomenological deformation nonlinear elastic-creeping body. The uniaxial stress - strain state of a mathematical expression without taking into account non force effects, this hypothesis is as follows: 
a) by the static effects

$$
\begin{aligned}
& \varepsilon\left(t, t_{0}\right)=\left[\frac{1}{E_{0}(t)}\right] \sigma(t) S_{m}\left[\frac{\sigma(t)}{R(t)}\right]+\sigma(t) S_{p}\left[\frac{\sigma(t)}{R(t)}\right] C\left(t, t_{0}\right)- \\
& -\int_{t_{0}}^{t} \sigma(\tau) S_{p}\left[\frac{\sigma(\tau)}{R(\tau)}\right] \frac{d}{d \tau} C(\tau, t) d \tau
\end{aligned}
$$

b) by the dynamic effects

$$
\begin{aligned}
& \varepsilon\left(t, t_{0}\right)=\left[\frac{1}{E_{0}(t)}\right] \sigma(t) S_{m}\left[\frac{\sigma(t)}{R(t)}\right]+\sigma(t) S_{p}\left[\frac{\sigma(t)}{R(t)}\right] K\left(\sigma_{\max }, \sigma_{\min }, \omega_{\min }, \omega, t\right) C\left(t, t_{0}\right)- \\
& -\int_{t_{0}}^{t} \sigma(\tau) S_{p}\left[\frac{\sigma(\tau)}{R(\tau)}\right] K\left(\sigma_{\max }, \sigma_{\min }, \omega_{\min }, \omega, \tau\right) \frac{d}{d \tau} C(\tau, t) d \tau
\end{aligned}
$$

where: $\mathrm{S}_{\mathrm{m}}$ and $\mathrm{S}_{\mathrm{p}}$ - nonlinearity function respectively instantaneous deformation and creep deformation; бand $\mathrm{R}$ - respectively stress and strength of the material; $\mathrm{E}_{0}$ - initial modulus of instantaneous deformation; $\mathrm{C}\left(\mathrm{t}_{0}, \mathrm{t}\right)$ - a simple measure of the initial creep; $\mathrm{t}_{0}$ - the startup; $\tau$ - the current time; $t$ - the end of the observation; $\left.K\left(\sigma_{\max }, \sigma_{\min }, \omega_{\min }, \omega, \tau\right)\right)$ - vibrocreep coefficient determined by a special technique.

The first term on the right is the sum of the relative instantaneous deformation, the second - relative deformations so-called "fast-leakage creep", the third - the relative lagging creep deformation accumulated over time. The function of non-linearity that is included in the equation $(6,7)$ are advised to take for instant strain:

$$
S_{m}=1+V_{m}\left(\frac{\sigma}{R}\right)^{m_{m}}
$$

for creep deformation:

$$
S_{p}=1+V_{p}\left(\frac{\sigma}{R}\right)^{m_{p}}
$$

The components of the previous dependence together with the equations (6 -9) we get:

$$
\begin{gathered}
\vec{\varepsilon}_{m}=\left[1+\vec{V}_{m}\left(\frac{\sigma}{R}\right)^{\vec{m}_{m}}\right] \frac{\sigma(t)}{\vec{E}_{m}^{0}(t)} ; \overleftarrow{\varepsilon}_{m}=\left[1+\overleftarrow{V}_{m}\left(\frac{\sigma}{R}\right)^{\bar{m}_{m}}\right] \frac{\sigma(t)}{\bar{E}_{m}^{0}(t)} ; \\
\left.\vec{\varepsilon}_{p}=-\int_{t_{0}}^{t}\left\{1+\vec{V}_{p}\left[\frac{\sigma(\tau)}{R(\tau)}\right]^{\vec{m}_{p}}\right\} \sigma(\tau) \frac{\partial}{\partial \tau} K\left(\sigma_{\max }, \sigma_{\min } \omega_{\min }, \omega, \tau\right) \vec{C}(t, \tau) \partial \tau ;\right\} \\
\bar{\varepsilon}_{p}=-\int_{t_{0}}^{t}\left\{1+\bar{V}_{p}\left[\frac{\sigma(\tau)}{R(\tau)}\right]^{\bar{m}_{p}}\right\} \sigma(\tau) \frac{\partial}{\partial \tau} K\left(\sigma_{\max }, \sigma_{\min } \omega_{\min }, \omega, \tau\right) \bar{C}(t, \tau) \partial \tau ;
\end{gathered}
$$

here: $\vec{V}_{m}$ and $\vec{m}_{m}$ respectively the mechanical characteristics of the nonlinear deformation when uploading to instantaneous deformation; $\bar{V}_{m}$ and $\bar{m}_{m}$-- the same - when unloading; 
$\vec{V}_{p}$ and $\vec{m}_{p}$, and $\quad \bar{V}_{p}$ and $\bar{m}_{p}$ similar characteristics to creep deformation; $\vec{E}_{0}(t)$ and $\quad \bar{E}_{0}(t)$ - initial modules instant deformations when loaded and unloaded; $K\left(\sigma_{\max }\right.$, $\left.\sigma_{\min }, \omega\right)$ - vibrocreep coefficient; $\vec{C}(t, \tau)$ and $\bar{C}(t, \tau)$ - initial steps simple creep.

Mechanical characteristics for the load quite reliably established on the basis of numerous experimental data, as for unloading is available at the moment not enough information to define them. Therefore they may be appointed in an indirect way on the basis of the hypothesis of central symmetry of the hysteresis loop, the mathematical interpretation of which is as follows:

$$
\left.\frac{d \vec{\varepsilon}}{d \sigma}\right|_{\sigma=0}=\left.\frac{d \bar{\varepsilon}}{d \sigma}\right|_{\sigma=\sigma_{\max }} ;\left.\frac{d \bar{\varepsilon}}{d \sigma}\right|_{\sigma=0}=\left.\frac{d \vec{\varepsilon}}{d \sigma}\right|_{\sigma=\sigma_{\max }} ;\left.\frac{\vec{\varepsilon}}{\sigma}\right|_{\sigma=\sigma_{\max }}=\left.\frac{\bar{\varepsilon}}{\sigma}\right|_{\sigma=\sigma_{\max }}
$$

It is proposed, based on the independence of the initial module unloading of the achieved level of the stress state, in order to further simplify, to believe in the equation (11) $\sigma_{\max }=\mathrm{R}$. It is necessary to pay attention to the following fact that the dynamic effects of materials strength of $\mathrm{R}$ is a variable the value of which depends on the coefficient of the oscillation cycle asymmetry determined on the basis of experimental data:

$$
R=R(\rho) ; \quad \rho=\text { omin/omax }
$$

Dependency (11) within one cycle of oscillation together with the dependency (10) within one cycle of oscillation andassumingvibrocreep in such a short time period almost does not develop and the transition to the new system, $\sigma$ coordinates $\sigma \mathrm{O}_{\varepsilon} \mathcal{\varepsilon}^{\mid}$(Fig. 1); offered the following values for the deformation characteristics:

$$
\begin{gathered}
\bar{m}_{m}=\frac{1}{\vec{m}_{m}} ; \quad \bar{m}_{p}=\frac{1}{\vec{m}_{p}} ; \quad \bar{V}_{m}=\frac{\vec{V}_{m} \vec{m}_{m}\left[\frac{\sigma_{\max }-\sigma_{\min }}{R(\rho)}\right]\left(m_{m}-\frac{1}{\vec{m}_{m}}\right)}{1+\left(1+\vec{m}_{m}\right) \vec{V}_{m}\left[\frac{\sigma_{\max }-\sigma_{\min }}{R(\rho)}\right]^{\vec{m}_{m}}} \\
\bar{V}_{p}=\frac{\vec{V}_{p} \vec{m}_{p}\left[\frac{\sigma_{\max }-\sigma_{\min }}{R(\rho)}\right]\left(m_{p}-\frac{1}{\vec{m}_{p}}\right)}{1+\left(1+\vec{m}_{p}\right) \vec{V}_{p}\left[\frac{\sigma_{\max }-\sigma_{\min }}{R(\rho)}\right]^{\vec{m}_{n}}} \\
\bar{E}_{m}=\frac{1+\left(1+\vec{m}_{m}\right) \vec{V}_{m}\left[\frac{\sigma_{\max }-\sigma_{\min }}{R(\rho)}\right]^{\vec{m}_{m}} \vec{E}_{m}^{0}}{1+\left(1+\vec{m}_{p}\right) \vec{V}_{p}\left[\frac{\sigma_{\max }-\sigma_{\min }}{R(\rho)}\right]^{\vec{m}_{m}} \vec{C}} .
\end{gathered}
$$

At long stationary vibration for concrete actions can be recommended to use a creep measure, and module parameters instantaneous deformation and nonlinearity, depending on the designing strength: 


$$
\begin{gathered}
C(t, \tau)=F_{n}(\tau) \vec{C}(\infty) f(t, \tau)+\frac{1}{\vec{E}_{m}^{0}(\tau)}-\frac{1}{\vec{E}_{m}^{0}(t)} \\
\vec{E}_{m}^{0}=4,55 \times 10^{4}-1,74 \times 10^{5} \frac{1}{R_{b}} ; \quad \vec{V}_{m}=\frac{37,5}{R_{b}} ; \quad \vec{V}_{n}=\frac{45}{R_{b}} ; \\
\vec{m}_{m}=5,7-0,05 R_{b} ; \quad \vec{m}_{n}=5-0,07 R_{b}
\end{gathered}
$$

where: $\mathrm{F}(\tau)$ - a function of age; $\mathrm{C}(\infty)$ - a measure of the ultimate creep of concrete; $\mathrm{Rb}$ - designing strength of concrete in MPa; $\mathrm{f}(\mathrm{t}, \tau)$ - function creep deformation of growth:

$$
f(t, \tau)=1-e^{-\gamma(t-\tau)} .
$$

To implement the proposed apparatus for calculating the coefficient vibrocreep proposed the following changes in the function of stress for prolonged harmonic or harmonic modes of approaching the dynamic effects on the stage of loading and unloading:

$$
\begin{gathered}
\vec{\sigma}(t)=\sigma_{\text {min }}+\left(\sigma_{\max }-\sigma_{\min }\right) \sin \frac{\omega\left(t-t_{0}\right)}{2} t_{0} \leq t \leq t_{0}+\frac{\pi}{\omega} \\
\bar{\sigma}(t)=\sigma_{\text {min }}+\left(\sigma_{\max }-\sigma_{\min }\right)\left[1+\cos \frac{\omega\left(t-t_{0}\right)}{2}\right]_{\text {для }} t_{0}+\frac{\pi}{\omega} \leq t \leq t_{0}+\frac{2 \pi}{\omega} ;
\end{gathered}
$$

Considering the dependence (10-13), together with (16) and omitting intermediate calculations, vibrocreep coefficient value can be recommended for use as follows:

$$
\mathrm{K}\left(\sigma_{\max }, \sigma_{\text {min }}, \omega, t_{0}\right)=\frac{G_{n o}\left(\sigma_{\max }, \sigma_{\text {min }} \omega_{\min }, t, \tau\right)}{G_{n o}\left(\sigma_{\max }, \sigma_{\min }, \omega, t, \tau\right)}
$$

where: $\omega_{\min }$ - the lowest oscillation frequency at which the material is trapped in the beginning of the manifestation vibrocreep for the materials in question. This value can be taken as 15-30 oscillations per minute, while taking into account the well-known link between the angular frequency of oscillations $(\omega)$ and the number of oscillations (n) can be obtained:

$\omega_{\min }=2 \times 3,14 \times 15 / 60 \ldots 2 \times 3,14 \times 30=(1,5 \ldots 3) 1 /$ second ;

$\mathrm{G}_{\mathrm{no}}$ - taking into account the consideration of only one cycle of the hysteresis process takes the following form:

$$
G_{n o}=\int_{t_{0}+\frac{\pi}{\omega}}^{t_{0}+\frac{2 \pi}{\omega}} \frac{d \bar{\sigma}}{d t} d t \int_{t_{0}}^{t} \bar{S}_{p} \frac{d}{d \tau} \bar{C} d t-\int_{t_{0}+\frac{\pi}{\omega}}^{t_{0}+\frac{2 \pi}{\omega}} \frac{d \vec{\sigma}}{d t} d t \int_{t_{0}}^{t} \vec{S}_{p} \frac{d}{d \tau} \vec{C} d t
$$

Substituting expression (19) from the stress values (16), we obtain: 


$$
\begin{aligned}
& G_{n o}=\int_{t_{0}}^{t_{0}+\frac{\pi}{\omega}}\left\{\sigma_{\min }+\left(\sigma_{\max }-\sigma_{\min }\right)\left[\sin \frac{\omega\left(t-t_{0}\right)}{2}\right]^{2}\right\} \frac{\partial}{\partial t} C d t- \\
& -\left(1+m_{n}\right) V_{n}\left[\frac{\sigma_{\max }-\sigma_{\min }}{R(\rho)}\right]^{m_{n} t_{0}+\frac{2 \pi}{\omega}} \int_{t_{0}+\frac{\pi}{\omega}}^{\omega}\left\{\sigma_{\min }+\left(\sigma_{\max }-\sigma_{\min }\right)\left[1+\cos \frac{\omega\left(t-t_{0}\right)}{2}\right]^{2}\right\} \frac{\partial}{\partial t} C d t+ \\
& +\frac{2 V_{n}}{2+m_{n}}\left[\frac{\sigma_{\max }-\sigma_{\min }}{R(\rho)}\right]^{m_{n} t_{0}+\frac{\pi}{\omega}} \int_{t_{0}}^{\frac{2}{\omega}}\left\{\sigma_{\min }+\left(\sigma_{\max }-\sigma_{\min }\right)\left[\sin \frac{\omega\left(t-t_{0}\right)}{2}\right]^{2+m_{n}}\right\} \frac{\partial}{\partial t} C d t- \\
& -\frac{2 V_{n} m_{n}}{2+\frac{1}{m_{n}}}\left[\frac{\sigma_{\max }-\sigma_{\min }}{R(\rho)}\right]^{m_{n}} \int_{t_{0}+\frac{2 \pi}{\omega}}^{\int_{t_{0}+\frac{\pi}{\omega}}^{\omega}}\left\{\sigma_{\min }+\left(\sigma_{\max }-\sigma_{\min }\right)\left[1+\cos \frac{\omega\left(t-t_{0}\right)}{2}\right]^{\left(2+\frac{1}{m_{n}}\right)}\right\} \frac{\partial}{\partial t} C d t
\end{aligned}
$$

For practical calculations proposed an interpolation method allowing you simply to determine the coefficient vibrocreep tabulated based on its values for different classes of concrete and load levels using the formula:

$$
\mathrm{K}_{\rho}=\rho-(1-\rho) K_{0}
$$

where: $\mathrm{K}$ - the coefficient table value vibrocreep.

It should be noted that these arguments are valid only for the case of uniaxial loaded elements, while in the practice of calculation and design of three-dimensional problems occur, or at least two-dimensional. However, the method of calculation referred to vibrocreep fairly easy to extend and triaxial stress - strain state. Considering the equations of Hooke's law with the three-dimensional stress state in the case of common nonlinear rheological deformation can be written:

$$
\begin{aligned}
& \varepsilon_{x}\left(t, t_{0}\right)=\frac{\sigma_{x}(t) S_{m}\left[\frac{\sigma_{\dot{*}}(t)}{R^{*}(\rho, t)}\right]-v\left\{\sigma_{y}(t) S_{m}\left[\frac{\sigma_{y}(t)}{R^{*}(\rho, t)}\right]+\sigma_{z}(t) S_{m}\left[\frac{\sigma_{z}(t)}{R^{*}(\rho, t)}\right]\right\}}{E_{0}(t)}+ \\
& +\left\{\sigma_{x}(t) S_{p}\left[\frac{\sigma_{x}(t)}{R^{*}(\rho, t)}\right] \mathrm{K}\left(\sigma_{x}, \omega_{\min }, \omega, t\right)-v\left(\begin{array}{c}
\sigma_{y}(t) S_{p}\left[\frac{\sigma_{y}(t)}{R^{*}(\rho, t)}\right] \mathrm{K}\left(\sigma_{y}, \omega_{\min }, \omega, t\right)+ \\
+\sigma_{z}(t) S_{p}\left[\frac{\sigma_{z}(t)}{R^{*}(\rho, t)}\right] \mathrm{K}\left(\sigma_{z}, \omega_{\min }, \omega, t\right)
\end{array}\right)\right\} C\left(t, t_{0}\right)- \\
& -\int_{t_{0}}^{t} \sigma_{x}(\tau) S_{p}\left[\frac{\sigma_{x}(\tau)}{R^{*}(\rho, \tau)}\right] K\left(\sigma_{x}, \omega_{\min }, \omega, \tau\right) \frac{\partial}{\partial \tau} C(\tau, t) d \tau+ \\
& \left.+v \int_{t_{0}}^{t} \sigma_{y}(\tau) S_{p}\left[\frac{\sigma_{y}(\tau)}{R^{*}(\rho, \tau)}\right] \mathrm{K}\left(\sigma_{y}, \omega_{\min }, \omega, \tau\right) \frac{\partial}{\partial \tau} C(\tau, t) d \tau+\right\} \\
& +v \int_{t_{0}}^{t} \sigma_{z}(\tau) S_{p}\left[\frac{\sigma_{z}(\tau)}{R^{*}(\rho, \tau)}\right] \mathrm{K}\left(\sigma_{z}, \omega_{\min }, \omega, \tau\right) \frac{\partial}{\partial \tau} C(\tau, t) d \tau
\end{aligned}
$$




$$
\begin{aligned}
& \gamma_{x y}=\frac{2(1+v)}{E_{0}(t)} \tau_{x y}(t) S_{m}\left[\frac{\tau_{x y}(t)}{R_{r}^{*}(\rho, t)}\right]+2(1+v) \tau_{x y} S_{m}\left[\frac{\tau_{x y}(t)}{R_{r}^{*}}\right] \mathrm{K}\left(\tau_{x y}, \omega_{\min }, \omega, y\right) C\left(t, t_{0}\right)- \\
& -2(1+v) \int_{t_{0}}^{t} \tau_{x y}(\tau) S_{p}\left[\frac{\tau_{x y}(\tau)}{R_{r}^{*}(\rho, \tau)}\right] \mathrm{K}\left(\tau_{x y}, \omega_{\min }, \omega, \tau\right) \frac{\partial}{\partial \tau} C(t, \tau) d \tau
\end{aligned}
$$

where:

$$
\mathrm{R}^{*}(\rho, \mathrm{t})=\mathrm{R}^{*}\left(\gamma_{\mathrm{b}}, \mathrm{t}\right)
$$

$\mathrm{R} *\left(\gamma_{\mathrm{b}}, \mathrm{t}\right)$ - generalized strength of concrete under dynamic loading, taking into account its decrease due to fatigue of material and to take concrete deformed under triaxial stress state in accordance with the theory of strength, depending on the characteristics of strength $\left(\mathrm{R}_{\mathrm{b}}\right.$ compressive and $\mathrm{R}_{\mathrm{bt}}$ - tensile) based on thetriaxial strength criterion in terms of volume deformation; $\gamma_{\mathrm{b}}$ - working conditions coefficient received on tabular data, depending on the stress ratios:

$$
\rho_{\mathrm{b}}=\sigma_{\min } / \sigma_{\max }=\left(\sigma_{\mathrm{st}}-\sigma_{\text {din }}\right) /\left(\sigma_{\mathrm{st}}+\sigma_{\mathrm{din}}\right)<1
$$

$\sigma_{\mathrm{st}}$ - stress in design element from the static component of the external load received from results of static analysis; $\sigma_{\mathrm{din}}$ - the same, from the effects of dynamic loading component.

Expressions (22) are greatly simplified in the case of quasi-linear creep, allowing you to significantly reduce the mathematical difficulties, in this case can be recommended for use as follows:

$$
\begin{gathered}
\varepsilon_{x}\left(t, t_{0}\right)=S\left[\frac{\sigma(t)}{R^{*}(\rho, t)}\right]\left\{\frac{1}{E_{0}(t)}\left[\sigma_{x}(t)\right]+\left[\sigma_{x}(t)\right] C\left(t, t_{0}\right)-\right. \\
\left.-\int_{t_{0}}^{t} \sigma_{x}(\tau) \mathbb{K}\left(\sigma_{x}, \omega_{\min }, \omega, \tau\right) \frac{\partial}{\partial \tau} C(\tau, t) d \tau\right\}+ \\
+S\left[\frac{\sigma(t)}{R^{*}(\rho, t)}\right]\left\{\frac{v}{E_{0}(t)}\left[\sigma_{y}(t)\right]+\left[\sigma_{y}(t)\right] C\left(t, t_{0}\right)-\right. \\
\left.v \int_{t_{0}}^{t} \sigma_{y}(\tau) K\left(\sigma_{y}, \omega_{\min }, \omega, \tau\right) \frac{\partial}{\partial \tau} C(\tau, t) d \tau\right\}+ \\
+S\left[\frac{\sigma(t)}{R^{*}(\rho, t)}\right]\left\{\frac{v}{E_{0}(t)}\left[\sigma_{z}(t)\right]+\left[\sigma_{z}(t)\right] C\left(t, t_{0}\right)-\right. \\
\left.v \int_{t_{0}}^{t} \sigma_{z}(\tau) K\left(\sigma_{z}, \omega_{\min }, \omega, \tau\right) \frac{\partial}{\partial \tau} C(\tau, t) d \tau\right\} \quad(25) \\
\gamma_{x y}=2(1+v) S\left[\frac{\tau_{x y}(t)}{R_{r}^{*}(\rho, t)}\right]\left\{\frac{\tau_{x y}(t)}{E_{0}(t)}+\tau_{x y} \mathrm{~K}\left(\tau_{x y}, \omega_{\min }, \omega, t\right) C\left(t, t_{0}\right)-\right. \\
-\int_{t_{0}}^{t} \tau_{x y}(\tau) \mathrm{K}\left(\tau_{x y}, \omega_{\min }, \omega, \tau\right) \frac{\partial}{\partial \tau} C(t, \tau) d \tau
\end{gathered}
$$

Note that according to $(22,25)$ the appointment of deformation nonlinearity parameter in determining the shear stresses should use experimental data results of shear tests and parameters to determine the non-linearity of the procedure on the basis of the decision of 
the logarithmic equation systems. However, due to some deficiency experimental data for all classes of concrete during shear deformation, especially in the nonlinear part, with a certain degree of approximation, we can permit the use of an axial test data. The value of the shear strength can be found from the known empirical formula:

$$
\mathrm{R}_{\mathrm{r}}^{*}(\rho, \mathrm{t})=0,7 \gamma_{\mathrm{b \tau}} \sqrt{ } \mathrm{R}_{\mathrm{b}}{ }^{*} \mathrm{R}_{\mathrm{bt}}^{*}
$$

here $\gamma_{b \tau}$ - the same ratio of working conditions, taking into account the reduction in strength due to concrete fatigue, received by the values of the coefficient of asymmetry of the cycle depends on the shear stresses:

$$
\rho_{\mathrm{b} \tau}=\tau_{\min } / \tau_{\max }=\left(\tau_{\mathrm{st}}-\tau_{\mathrm{din}}\right) /\left(\tau_{\mathrm{st}}+\tau_{\mathrm{din}}\right)<1
$$

Equations (22) and (25) can be used only in the case of so-called simple (simultaneous) loading at which the stress ratio in the XYZ axes remain constant. This assumption, which implies simultaneous compliance tensors of stresses and strains are well supported by the majority of experimental studies, but mainly for metals and their alloys. For concrete, even in the case of simple loading into force manifestations of nonlinearity of deformation takes place the well-known process of redistribution of forces and stresses between the individual elements of structures, between the components of the cross sections, cross sections within them, which leads to disruption of synchrony and turns even simple uploading to the complex. However, a well-known fact that the degree of violation of said synchronism with smooth changes in external loading mode or the steady dynamic oscillation mode is still little effect on the final outcome, and therefore assuming a certain degree of error of this factor can be ignored.In addition, the equation (22) and (25) can be used for the case only when the value of the coefficient of transverse deformation continuously on all axes, which is not until the end of a fair and evidenced by experimental studies, as well as the obvious fact that the concrete should not be considered strictly orthotropic material. The development over time of concrete transverse strains has not been studied. Available data and numerical values are often contradictory and have a large spread. Some of the data obtained from the results of experimental and research show that with increasing stress level increases the coefficients of relative transverse strains, these coefficients for elastically instant greater strain coefficients for creep, and the load they represent only $80 \%$ of the corresponding values for unloading. With the growing strength of the concrete crossstrain rate decreases. Therefore, strictly assess the qualitative and quantitative change in Poisson's ratio in a three-dimensional deformation at the present level of available results, at present, in our view, is not possible because of the large variation and inconsistencies in the data. In addition to the above it should be noted that taking into account the variable coefficient value of transverse strain in a triaxial deformation, while taking into account the rheological and nonlinear processes lead to considerable complication of governing equations, insuperable causing mathematical difficulties that are hard to overcome even with recommended linearizing apparatus integral estimates. It should be noted that taking into account the variable value of the coefficient of transverse deformation was realized for the plane strain conditions. Thus, the tradition, and most of the existing research rather authoritative authors devoted to consideration of triaxial stress-strain state of reinforced concrete structures suggest that the relative values of the coefficient of transverse strain, with some degree of permissible error can be considered constant. That of course does not rule out further research to clarify the laws change transverse strains, which will clarify the existing method of calculation in the longer term.

So, on the basis of the above, with some degree of approximation, can take several values averaged relative ratio of transverse strain, in particular for concrete, the hardening process of which can be assumed not ended $v=0,25$, while for the old concrete $v=0,2$ for 
reinforcement can be considered $v=0.3$. Such an assumption, though will lead to some error, but in terms of building dynamic analysis techniques can significantly facilitate the mathematical apparatus and obtain engineering foreseeable results.

\section{Conclusions}

Thus, the dynamic analysis of reinforced concrete structures in the conditions of triaxial stress state becomes much more complicated, since to determine the coefficient vibrocreep on three coordinate axes must be calculating the six components of normal and shear stresses. These difficulties are further compounded by the need to consider the creep deformation and nonlinearity. Note the additional complexity when performing dynamic analysis, since at each step of the iterative process for calculating the search unknowns should further take into account the dependence of concrete strength by the magnitude of the current dynamic stresses (or the asymmetry coefficient cycles) based on the triaxial strength criterion in terms of volume deformation. Such complexity of the mathematical apparatus can be successfully overcome and obtain the desired result with the help of linearization based on the method of integral estimates, including the fixation of the creep processes in the varied stresses at the material time of observation, as well as the use iterative processes in the implementation of the finite element method for solving engineering problems.

\section{References}

1. V. M. Bondarenko, V.I. Rimshin V. M. The theory of dissipative power resistance of reinforced concrete, 287 (2015)

2. E. M. Zverjaev, M. V. Berlinov, M. N. Berlinova. International Journal of Applied Engineering Research, 11(8), 5811 (2016)

3. E. A. Korol, M. V. Berlinov, M.N. Berlinova, Procedia Engineering, 165, 292 (2016)

4. E.A. Korol, Yu.A. Kharkin, Book of Reports of XX Russian-Polish-Slovak Workshop "Theoretical Fundamentals of Construction" 401 (2011)

5. V. M. Bondarenko,V.I.Kolchunov. Calculation models of the power resistance of reinforced concrete, 112 (2004)

6. M.N. Berlinova, A.V. Tvorogov. Natural and engineering sciences, 6 (84), 530 (2015) 